\title{
OPEN Dynamic mode decomposition of inertial particle caustics in Taylor-Green flow
}

\author{
Omstavan Samant ${ }^{1}$, Jaya Kumar Alageshan ${ }^{2 \varpi}$, Sarveshwar Sharma ${ }^{3}$ \& Animesh Kuley ${ }^{2}$ \\ Inertial particles advected by a background flow can show complex structures. We consider inertial \\ particles in a 2D Taylor-Green (TG) flow and characterize particle dynamics as a function of the \\ particle's Stokes number using dynamic mode decomposition (DMD) method from particle image \\ velocimetry (PIV) like-data. We observe the formation of caustic structures and analyze them using \\ DMD to (a) determine the Stokes number of the particles, and (b) estimate the particle Stokes number \\ composition. Our analysis in this idealized flow will provide useful insight to analyze inertial particles \\ in more complex or turbulent flows. We propose that the DMD technique can be used to perform \\ similar analysis on an experimental system.
}

Advection of particles, such as dust or aerosol by a background flow is a ubiquitous phenomena. And the study of dispersion of these inertial particles are of immense interest both for applied and natural processes, in particular, to analyze oil spills in oceans ${ }^{1-4}$, dispersion of pollutants and toxic elements ${ }^{5,6}$, suspended particles in aquatic systems $s^{7}$, formation of clouds ${ }^{8,9}$ and volcanic plumes ${ }^{10}$, and the effect of the flow patterns generated by the breathing action and cough on the dispersion of the aerosol particles are crucial to understand the spread of COVID-19 virus ${ }^{11-16}$. Numerical studies of inertial particle dispersion in different types of flows, ranging from static $^{17}$ to turbulent ${ }^{18,19}$ flows have shown that the particles display complex dynamical behaviours like formation of fractal clusters ${ }^{18}$ and caustics ${ }^{20}$. The analysis of the structures formed by the particles encode information about the Stokes number of the particles and the flow patterns.

Experimental techniques such as particle image velocimetry (PIV) have been used to track particles and extract velocity profiles ${ }^{21}$ when it is possible to identify individual particles in an image, but not with certainty to track it between images. If the particle concentration is so low that it is possible to follow an individual particle it is called particle tracking velocimetry (PTV). While similar techniques have been adopted to track particles in simulations, the averaging process reduces the spatial resolution, which is critical in our application. In simulations, the Osiptov's method ${ }^{22}$ have been used to extract caustic features ${ }^{20}$, which track each particle in PTV like situations but fail for PIV like data. We propose the use dynamical mode decomposition (DMD) based scheme to obtain the spatio-temporal particle distributions as a representative for particle density. DMD methods have been used to extract coherent structures in simulations and experiments ${ }^{23-26}$ of fluids. We use the DMD method to analyze and extract the features of the caustics to (a) determine the Stokes number of the particles, and (b) estimate the relative particle concentrations in a bi-disperse Stokes number system. Our approach can also be extended to multiple Stokes number poly-disperse systems.

This paper is organized as follows. In "Model" we present the form of the TG flow, minimal model of an inertial particle, and the relevant numerical simulation details. In "Observations" we show the formation of caustic structures and analyze them using DMD method in "Analysis" and demonstrate how we extract the caustic wavefronts from the DMD mode. We use the position and the gradient of the wavefront in the DMD eigen mode to estimate the Stokes number and the composition of a bi-disperse Stokes number systems in "Determination of Stokes number from DMD" and "Estimation of particle concentration" respectively, and present our conclusions in "Conclusions".

\footnotetext{
${ }^{1}$ Centre for Fusion, Space and Astrophysics, University of Warwick, Coventry CV4 7AL, UK. ${ }^{2}$ Department of Physics, Indian Institute of Science, Bangalore 560012, India. ${ }^{3}$ Institute For Plasma Research, Gandhinagar, Gujarat 382428, India. ${ }^{\circledR}$ email: jkumar.res@gmail.com
} 


\section{Model}

We consider a 2D lattice of vortices in the form of a Taylor-Green (TG) flow. The TG flow is a steady state solution to the forced, incompressible Navier-Stokes equation and can be considered a convection model in $2 \mathrm{D}^{27,28}$. Such a flow can be experimentally setup using ion solutions in an array of magnets ${ }^{29}$. The TG flow is given by the vorticity field as

$$
\omega(x, y)=\omega_{0} \sin \left(\frac{2 \pi x}{L}\right) \sin \left(\frac{2 \pi y}{L}\right)
$$

and the corresponding velocity field is

$$
\mathbf{u}(x, y)=V_{0}\left[\begin{array}{rr}
\sin \left(\frac{2 \pi x}{L}\right) & \cos \left(\frac{2 \pi y}{L}\right) \\
-\cos \left(\frac{2 \pi x}{L}\right) & \sin \left(\frac{2 \pi y}{L}\right)
\end{array}\right]
$$

where $x, y \in[0, L)$ and are periodic, $\mathbf{u}$ is the Eulerian velocity field such that $\omega=\nabla \times \mathbf{u}$, with $\omega_{0}=4 \pi V_{0} / L$. We choose $V_{0}$ as the velocity scale and $L$ as the length scale and write the system parameters in corresponding dimensionless form. We model the aerosol particles as small rigid spheres, which are effectively points, that have density different from the surrounding fluid. The equation of motion of the inertial particles in a background flow given by the simplified Maxey-Riley approximation ${ }^{17}$ for small particles that are much denser than the fluid are

$$
\begin{aligned}
& \frac{d \mathbf{x}}{d t}=\mathbf{v} \\
& \frac{d \mathbf{v}}{d t}=\frac{1}{S t}(\mathbf{u}-\mathbf{v})
\end{aligned}
$$

where $S t$ is the Stokes number which captures the effect of particle inertia, $\mathbf{x}$ is the particle position and $\mathbf{v}$ is the particle velocity (see Appendix A for validity of the equations). The case when $S t \rightarrow 0$ the particles act as tracers that follow the velocity stream lines and the Eq. (3) leads to $\mathbf{v}=\mathbf{u}$. We use RK4 numerical scheme to discretize and evolve the particle positions and velocities. Furthermore, we use periodic boundary conditions, such that the particles are reintroduced into the system when they exit the boundary. In our analysis we set the time step to $\Delta t=0.01\left(L / V_{0}\right)$.

\section{Results}

Observations. A typical feature of inertial particles in a background flow is that they are expelled from high vorticity regions. In a background flow with vortices, the inertial particles can form caustics ${ }^{20}$. So in the case of TG flow, the inertial particles tend to accumulate along the low vorticity regions that separate the vortices. We simulate the dynamics of inertial particles in a TG flow and observe spatial regions where the Lagrangian velocity field is multi-valued, which are referred to as caustics ${ }^{30-32}$ (see Fig. 3a).

Figure 1 shows the caustic structure formed by a mono-disperse inertial particle system, when we start the simulation with the particle positions initialized with uniform random distribution within the $L \times L$ box, and setting the initial velocities of the particles to zero. In the long time limit, the particles accumulate along a curve $\mathrm{e}^{33}$. We observe that the caustics that form in the transient state are robust and stable to small perturbations (see Appendix A), whereas the steady state structures break up and lead to chaos ${ }^{33}$. Furthermore, the steady state behaviour strongly depends on the system size and the boundary conditions.

We find that for a range of Stokes numbers the caustic structures preserve their shapes; and their sizes depend on St. In the following section we use dynamic mode decomposition (DMD) to extract features of the structures, in particular the caustic wavefront, and study its size dependence on the Stokes number. Furthermore, the sharpness of the caustic wavefront enables us to detect and extract their sizes even in presence of poly-disperse Stokes number systems.

Analysis. The caustics in Fig. 1 have a complex structure and in the presence of multiple Stokes number particles resolving these structures from a single snap-shot is hard. Therefore we employ the spatio-temporal data in the form of a video sequence that contains $\mathscr{F}$ frames of $N \times N$ pixel images and analyze them using the dynamic mode decomposition (DMD) method.

DMD is a data analysis technique that has been used to extract coherent structures in fluid dynamic systems ${ }^{34}$, where it is able to extract different modes that are similar to normal modes in linear dynamical systems. The $\mathrm{DMD}$ is a data-driven technique introduced by Schmid as a numerical procedure for extracting dynamical features from flow data ${ }^{24}$. The DMD algorithm takes in a time-series data in the form of vectors $\left\{\mathbf{v}_{1}, \mathbf{v}_{2}, \ldots \mathbf{v}_{T}\right\}$ and estimates a linear dynamical system that can generate a map

$$
\mathbf{v}_{i+1}=\mathscr{A} \mathbf{v}_{i}
$$

where $\mathscr{A}$ is an $N^{2} \times N^{2}$ matrix and the eigenvectors of $\mathscr{A}$ form the DMD modes, with the corresponding eigenvalues. Finally, the eigenvectors are reshaped into $N \times N$ pixel image to obtain the modes. A Singular Value Decomposition (SVD) based algorithm for estimating the DMD modes is described in Appendix B.

Let $i$ stand for the iteration number such that the particles are in their stationary initial state and start their evolution at $i=0$. Then for our data, the vectors $v_{i}$ are obtained by rearranging the $N \times N$ pixel images at instant $i$ into $N^{2} \times 1$ vector. In our DMD analysis we employ $\left\{v_{250}, v_{251}, \ldots v_{750}\right\}$ (i.e. $\mathscr{F}=500$ ), as the modes obtained 


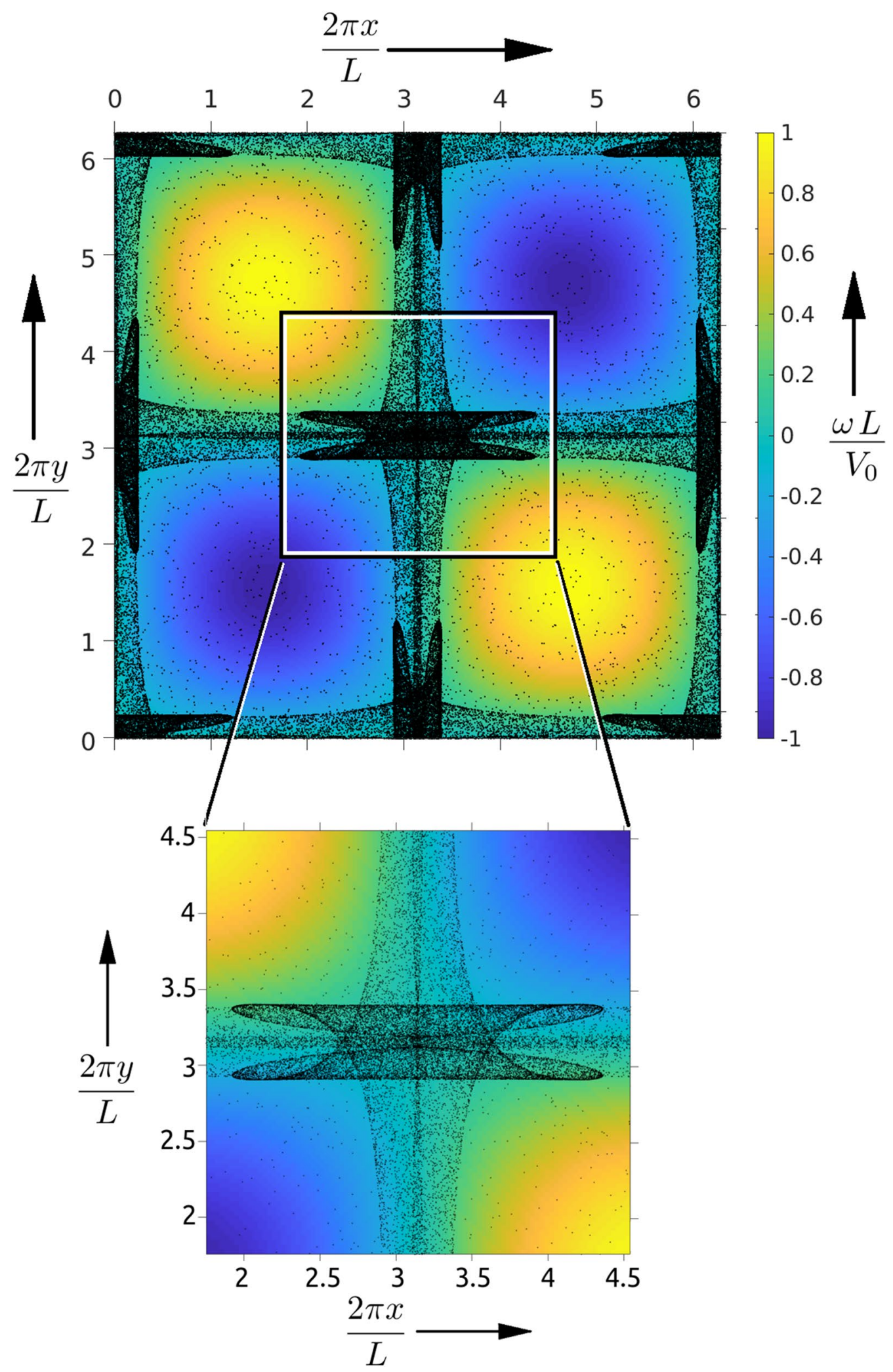

Figure 1. A snapshot of the $2 \mathrm{D}$ spatial particle distribution in (a) at $t=750 \Delta t$ shows the complex structure formed by the mono-dispersed inertial particles with $S t=1.0$ in a periodic domain of size $L \times L$. The background color-map corresponds to the vorticity field (with values given by the colorbar on the left side of the top panel) and the particles are plotted using black markers. Notice that the particles in the high vorticity regions have moved out towards the regions bounding the vortices. The zoomed-in version in (b) shows the details of the caustic structures around the central region of the domain. See the video C.1 for the evolution of the caustic structures. The figures were generated using Matlab 2020a, https://www.mathworks.com/products/ matlab.html. 

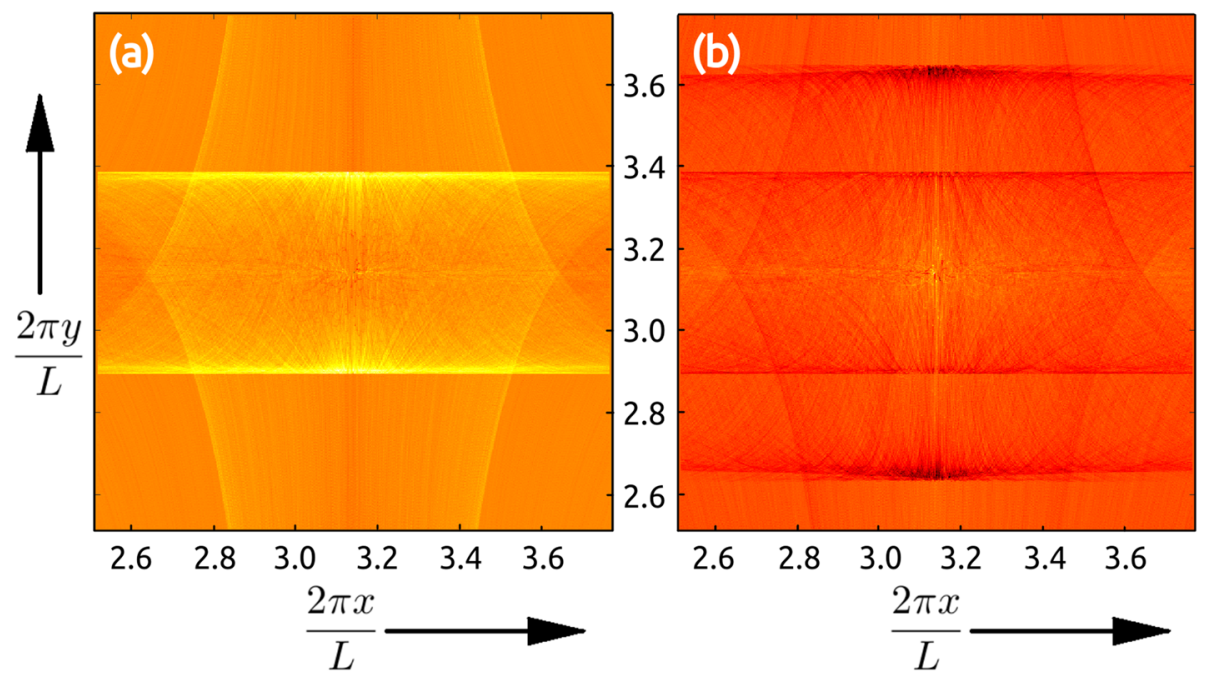

Figure 2. The highest singular DMD eigenvector, $\mathscr{D}{ }^{(1)}$, obtained for: (a) $S t=1.0$ mono-disperse system, (b) a bi-disperse mixture of $S t=\{1,2\}$ with 7:3 ratio of initial particle concentrations. The horizontal lines correspond to the caustic wavefronts, and the number of such fronts indicate the different Stokes number particles. Also notice that the wavefronts corresponding to $S t=1.0$ appear around the same values of $y$ in both $(\mathbf{a}, \mathbf{b})$, indicating that the positions of DMD wavefronts are not perturbed by the presence of particles with different Stokes numbers. The figures were created using Matlab 2020a, https://www.mathworks.com/products/ matlab.html.
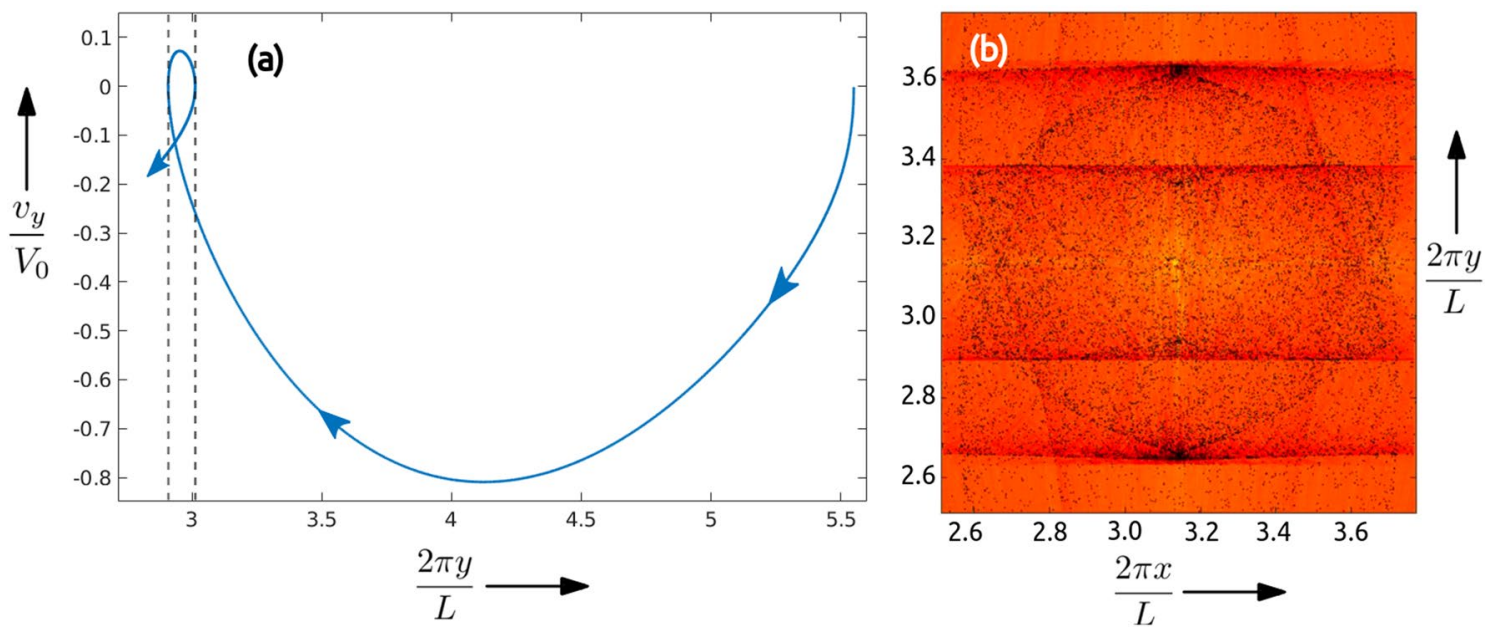

Figure 3. (a) The particle trajectory in the reduced phase space, $\left(y, v_{y}\right)$, shows the multi-valued nature of the casutics in velocity between the dotted vertical lines ${ }^{32}$. The movie in C.2 shows the evolution of the bi-disperse particles overlaid on the corresponding DMD from Fig. $2 \mathrm{~b}$ and the plot (b) shows a snap-shot at the 650th iteration step when the caustics and the DMD wavefronts coincide. Notice that the first DMD picks out only the slow moving horizontal caustics. The plot and the figure was generated using Matlab 2020a, https://www.mathw orks.com/products/matlab.html.

are the sharpest for this range. Let $\mathscr{D}{ }^{(\alpha)}(j, k)$ represent the $(j, k)$ th pixel of the $\alpha$ th eigenmode, ordered in terms of decreasing absolute eigenvalue. Since the caustics are localized around the central region of the domain, we use a zoomed-in region of size $512 \times 512$ pixels (i.e. $N=512$ ) in our analysis, as shown in Fig. 1 . We find that the highest singular eigenvalue mode, namely $\mathscr{D}^{(1)}$, shown in Fig. 2a highlights a straight-line caustic structure, which we refer to as the wavefront. The eigenvalues of other DMD modes decay exponentially. We observe that the position of the wavefront in $\mathscr{D}^{(1)}$ has a systematic dependence on the Stokes number, and to extract this relation we detect the location of the wavefronts using edge detection techniques. Similarly, when we perform DMD analysis on a bi-disperse system $\mathscr{D}^{(1)}(j, k)$ shows two distinct wavefronts (see Fig. $2 \mathrm{~b}$ ) corresponding to the two different Stokes numbers; and here DMD uses the velocity information to unambiguously extract the wave fronts. In particular, Fig. 3a shows the reduced phase space portrait of a typical particle which form the caustic ${ }^{32}$ and Fig. $3 \mathrm{~b}$ shows the particles overlaid on top of the DMD that demonstrates the DMD's ability to extract the 

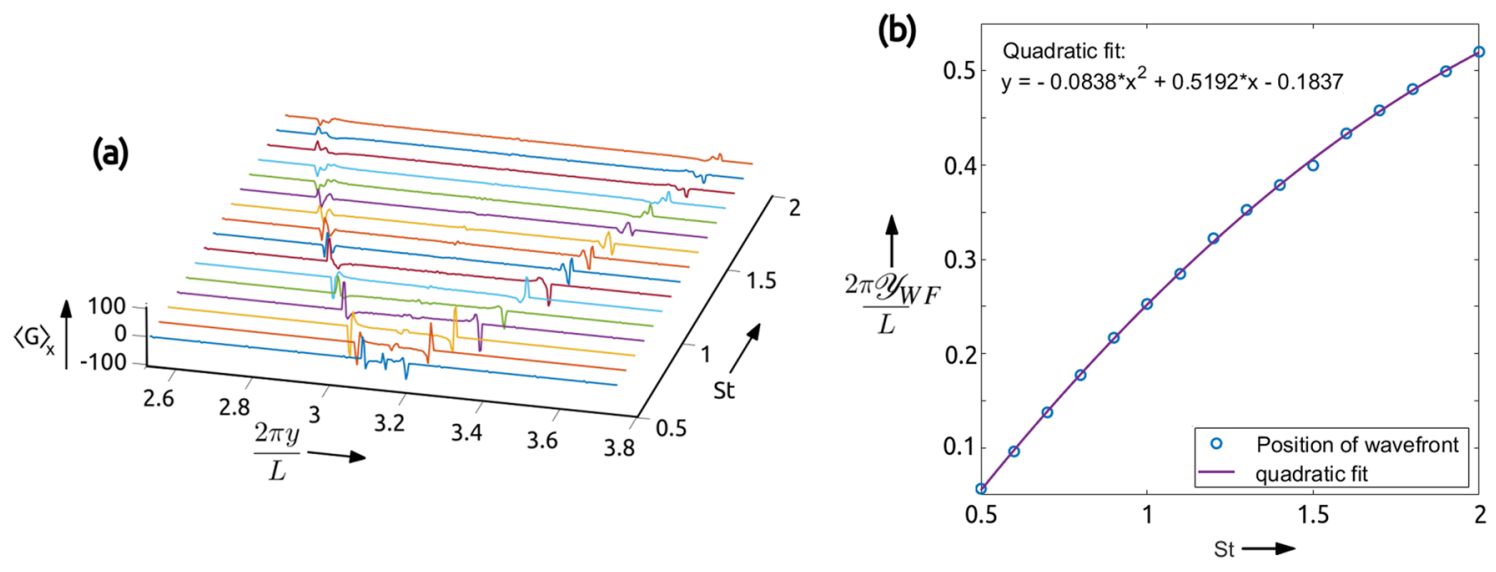

Figure 4. In (a) we show the plots of $\langle G\rangle_{x}$ obtained for different values of the Stokes number from monodisperse systems. We extract the location of the peaks in $\langle G\rangle_{x}$ for each $S t$, as defined in Eq. (7), to generate the plot in (b) and we find that the $\mathscr{Y}_{W F}$ and $S t$ have a quadratic dependence. The plots were generated using Matlab 2020a, https://www.mathworks.com/products/matlab.html.

caustic structures. Furthermore, we find that the intensity of each wavefront compared to the background, which we refer to as prominence ${ }^{35}$, depends on the corresponding initial particle concentrations in the system.

We now prescribe a method to extract the position of the wavefront from DMD. We use a Sobel operator ${ }^{36}$ such that the vertical gradient of the first DMD mode is given by

$$
G(j, k)=\frac{\partial \mathscr{D}^{(1)}(j, k)}{\partial y} \approx \frac{1}{\Delta y}\left[\begin{array}{ccc}
1 & 2 & 1 \\
0 & 0 & 0 \\
-1 & -2 & -1
\end{array}\right] \circledast \mathscr{D}^{(1)}(j, k)
$$

where $\circledast$ represents the $2 \mathrm{D}$ convolution operator ${ }^{37}$, and $\Delta y$ is the spacing in DMD along the $y$-axis. We then sum over the values in the $\mathrm{x}$-direction to get a $1 \mathrm{D}$ function of $\mathrm{y}$ as

$$
\langle G\rangle_{x}=\int_{0}^{L} G(j, k) d x \approx \sum_{j=1}^{N} G(j, k) \Delta x
$$

where $\Delta x$ is the spacing in DMD along the $x$-axis, and we choose a square grid with $\Delta x=\Delta y$ such that $\langle G\rangle_{x}$ is by definition independent of the grid spacing and is dimensionless. In the next section we describe how the location and the value of the peaks in $\langle G\rangle_{x}$ can be used to find the Stokes number of the particles and the relative initial concentrations in the case of a bi-disperse system.

Determination of Stokes number from DMD. The $\langle G\rangle_{x}$ is obtained from the DMD as described in "Analysis" by simulating mono-disperse Stokes number particle systems to generate the plots in Fig. 4a which shows $\langle G\rangle_{x}$ as a function of $S t$. The alignment of the peaks in $\langle G\rangle_{x}$ along a curve indicates a systematic dependence of the location of wavefront on the Stokes number. To extract this relation we first get the location of the caustic wavefront from the domain center using the position of the peaks in $\langle G\rangle_{x}$ given by

$$
\mathscr{Y}_{W F}=\arg \max _{y}\langle G\rangle_{x}-\frac{L}{2}
$$

where $\arg \max _{y}$ gives the value of $y$ for which $\langle G\rangle_{x}$ is maximum. We then plot $\mathscr{Y}_{W F}$ as a function of St as shown in Fig. 4b. Using a non-linear least squares fit method we find that the relation is of the form $\mathscr{Y}_{W F} \sim a S t^{2}+b S t+c$, with values of the parameters $a, b$, and $c$ as indicated in Fig. $4 \mathrm{~b}$, where $x$ represents $S t$. Now, extrapolating the fit we find that $\mathscr{Y}_{W F}=0$ at $S t=0.3767$ and becomes multi-valued for $S t>3.0979$, thus setting the limits on the validity of the relation. In stagnation-point flows below a certain value of Stokes number normally there are no caustics, as shown by Healy et al. ${ }^{22}$; the lower cut-off for the Stokes number in our case could be indicative of a similar phenomenon. The fact that $\mathrm{St}=0.37$ appears as a hard cut-off for caustics could be circumstantial and tied to the choice of initial condition and flows can actually display caustics at any Stokes number. Furthermore, we restrict our study to $S t<3$ motivated by similar studies in literature ${ }^{33}$. As described in Cencini et al. ${ }^{38}$, the Maxey-Riley approximation in Eq. (3) is not valid for Stokes numbers greater than 3 as the back-reaction of the particle on the flow becomes important. The three parameters in the relation can be estimated experimentally using calibrated measurements and the relation can be used to predict the St of new particle systems. In particular we demonstrate that the above method can be generalized to work in case of bi-disperse system.

As shown in Fig. 2b, for a bi-disperse system the DMD has two sets of caustic wavefronts, corresponding to each Stokes number. Now we set one of the Stokes number fixed at one $\left(S t_{1}=1.0\right)$, vary the $S t_{2}$ and find $\langle G\rangle_{x}$ to generate the plots in Fig. 5. The results in Fig. 5 show that even in the bi-disperse system the caustic wavefront has the same characteristic behaviour on the Stokes number as the mono-disperse system. In particular, the wavefront corresponding to $S t_{1}$ has a fixed location and the wavefront due to $S t_{2}$ preserves the dependence on 
$\langle G\rangle_{x}$

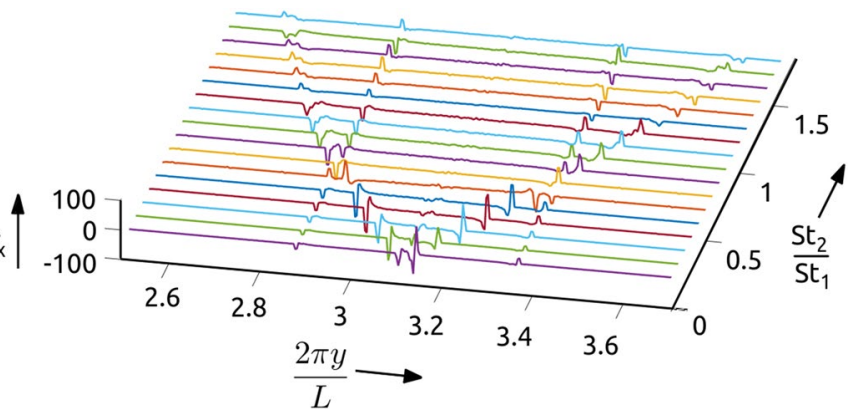

Figure 5. Plots of $\langle G\rangle_{x}$ for a bi-disperse system as a function of $y$ and the ratio of the the two particle Stokes numbers, with one of the Stokes number fixed at one $\left(S t_{1}=1.0\right)$, and varying $S t_{2}$. Notice that the peaks in $\langle G\rangle_{x}$ corresponding to $S t_{1}$ are aligned at the same location along $y$, whereas the peaks due to $S t_{2}$ show similar trend as the plots for mono-disperse systems in Fig. 4a. This plot was generated in Matlab 2020a, https://www.mathw orks.com/products/matlab.html.
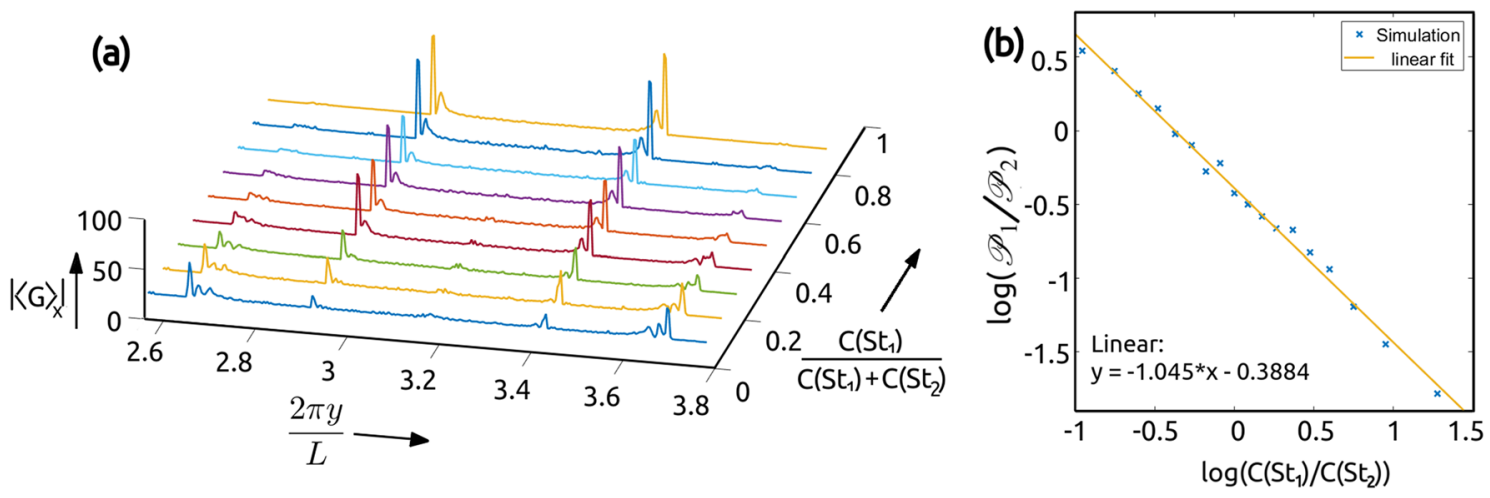

Figure 6. The plot (a) shows the variation in the absolute value of $\langle G\rangle_{x}$ for a bi-disperse system, with $S t_{1}=1$ and $S t_{2}=2$ particles, for various initial concentration fraction $C\left(S t_{1}\right) /\left(C\left(S t_{1}\right)+C\left(S t_{2}\right)\right)$. Notice that the peaks corresponding to each wavefront is not unique and have a finite spread in $\mathrm{y}$. In (b) the relation between the prominence corresponding to $S t_{1}$ and $S t_{2}$ are given by $\mathscr{P}_{1}, \mathscr{P}_{2}$ respectively, as a function of the initial particle concentrations $C$ is shown in a log-log plot. The linear fit shows that the ratios of the peaks of $|\langle\hat{G}\rangle|$ and the ratios of the concentration are related by a power-law, with a power close to -1 . The plots were generated using Matlab 2020a, https://www.mathworks.com/products/matlab.html.

$\mathscr{Y}_{W F}$ of the mono-disperse system. Our studies with poly-disperse $S t$ systems show that the caustic wavefronts can be used to find the Stokes number of different particles in the system using the relation obtained from a mono-disperse system.

Estimation of particle concentration. Until now the bi-disperse systems that we considered had equal number of $S t_{1}$ and $S t_{2}$ particles, with uniform initial distribution in space. Now we study the variation in $\mathscr{D}{ }^{(1)}$ w.r.t. the change in relative number of particles. We observe that the intensity of the wavefront or the gradient in the DMD image depends on the number of particles or the initial uniform concentration, denoted by $C(S t)$.

The variable $\langle G\rangle_{x}$ gives the gradient of $\mathscr{D}{ }^{(1)}$ along the vertical direction and the magnitude of the gradient indicates sharpness of the wavefronts (see Fig. 6a). To measure the sharpness of the wavefront we define a "prominence" parameter, $\mathscr{P}$, as the sum of the non zero values of $\left|\langle\bar{G}\rangle_{x}\right|$ in the neighbourhood of the wavefront, which takes into account multiple peaks in the vicinity of the wavefront. We find that the prominence of the wavefront has a systematic dependence on the initial concentration of the corresponding Stokes number particles and from Fig. $6 \mathrm{~b}$ we find that on a log-log plot the relation is linear, with a slope approximately equal to -1 . This implies that in a bi-disperse system the ratio of the prominence is inversely related to the ratio of initial concentrations. We can use this relation to predict the concentration of various Stokes number particles in a system.

\section{Conclusions}

We study the dynamics of inertial particles in a Taylor-Green flow with periodic boundary conditions in 2D. In a minimal model of inertial particles we observe that for a mono-disperse Stokes number system, starting from a uniform distribution of stationary particles, the particle distribution forms caustics in the strain dominated region of the flow. We use the DMD method to analyze the PIV-like time-series data of the spatio-temporal particle distribution and find that the largest absolute eigenvalue mode is effective in extracting the caustic wavefrontlike structure. We notice that (a) the position of the wavefront depends on the particle Stokes number and employ 
standard image processing techniques to quantitatively extract a quadratic relation. Using this relation we can predict the Stokes number from the wavefront position. Furthermore, we find that for a bi-disperse system the DMD is able to extract two different wavefronts corresponding to each Stokes number and the positions of each wavefront follow the same quadratic relation as in the case of mono-disperse system. We also observe that (b) the sharpness of the wavefront in the DMD, measured in terms of prominence, depends on the initial particle concentration and find that for a bi-disperse Stokes number system the ratio of the wavefront prominence is inversely proportional to the corresponding Stokes number initial concentration. Hence the measurement of prominence can be used to estimate the concentration of the corresponding Stokes number particles.

We propose that the DMD technique can be used to analyze real experimental PIV data of caustics and perform similar analysis to extract information about the Stokes numbers and concentrations of the particles. In future we will consider detailed Navier-Stokes equation for the self-consistent evolution of the velocities and analyze the caustic structures in $3 \mathrm{D}^{39}$ and turbulent flows ${ }^{18,40}$. Contrary to the Taylor-Green flow case, a single dominant DMD mode does not capture the complete features of the caustics in a turbulent flow. As described in Marensi et al. ${ }^{41}$, symmetries play an important role in data driven modal expansions for turbulent flows and would entail the use of symmetry-reduced dynamic mode decomposition (SRDMD).

\section{A Validity of particle dynamics}

The particle dynamics we use in our study is a special case of ${ }^{17}$

$$
\begin{aligned}
& \frac{d \mathbf{x}}{d t}=\mathbf{v} \\
& \frac{d \mathbf{v}}{d t}=\frac{1}{S t}(\mathbf{u}-\mathbf{v})+R\left(\mathbf{u} \cdot \nabla \mathbf{u}+\frac{1}{2} \mathbf{v} \cdot \nabla \mathbf{u}\right)+\eta(\mathbf{v}, t)
\end{aligned}
$$

where $\eta$ is the white noise and $R=\rho_{f} /\left(\rho_{p}+\rho_{f} / 2\right)$ for density of particle $\rho_{p}$ and density of fluid $\rho_{f}$. We present our results for $\eta=0$ and $R=0$, and use small values of these parameters to verify the stability of our results. In Wang et al. ${ }^{33}$ the authors show that for $R=0$ the system is not chaotic. It will be interesting to check for $R>0$ if the DMD modes can capture the transition of the system from periodic to chaotic states.

\section{B DMD algorithm}

The $N \times N$ pixel image at $k$ th instant, $I^{k}(i, j)$, is rearranged into an $N^{2} \times 1$ vector $X^{k}(m)$ (note that we subtract the mean value from $X^{k}(m)$ ). Now the $\mathscr{F}$ sequence of image frames are appended together to form $N^{2} \times N_{T}$ matrix $Y$. Let the $N^{2} \times(\mathscr{F}-1)$ dimensional matrix formed from the first $(\mathscr{F}-1)$ frames be $Y_{1}$ and the last $(\mathscr{F}-1)$ frames be $Y_{2}$, i.e.

$$
\begin{aligned}
{\left[I^{k}\right]_{N \times N} } & \longrightarrow\left[X^{k}\right]_{N^{2} \times 1} \\
{\left[X^{1}\left|X^{2}\right| \ldots \mid X^{N_{T}}\right] } & \longrightarrow[Y]_{N^{2} \times N_{T}} \\
{\left[X^{1}\left|X^{2}\right| \ldots \mid X^{\left(N_{T}-1\right)}\right] } & \longrightarrow\left[Y_{1}\right]_{N^{2} \times\left(N_{T}-1\right)} \\
{\left[X^{2}\left|X^{3}\right| \ldots \mid X^{N_{T}}\right] } & \longrightarrow\left[Y_{2}\right]_{N^{2} \times\left(N_{T}-1\right)}
\end{aligned}
$$

We find the singular value decomposition (SVD) of the matrix $Y_{1}$, such that $Y_{1}=U \Sigma V^{*}$, where $U$ is a $N^{2} \times N^{2}$ complex unitary matrix, $\Sigma$ is an $N^{2} \times(\mathscr{F}-1)$ rectangular diagonal matrix with non-negative real numbers on the diagonal, and $V$ is a $(\mathscr{F}-1) \times(\mathscr{F}-1)$ real or complex unitary matrix.

Now choose a lower dimensional SVD matrices made up of first $n_{T}(<<\mathscr{F})$ columns, represented by $\tilde{U}, \tilde{V}$, and the first $n_{T} \times n_{T}$ block of $\Sigma$ as $\tilde{\Sigma}$. Define a matrix $n_{T} \times n_{T}$ matrix $A$ as

$$
A=\tilde{U}^{*} Y_{2} \tilde{V} \tilde{\Sigma}^{-1}
$$

Then the dynamic modes are the $N^{2} \times 1$ eigenvectors of matrix $A$ that is rearranged into $N \times N$ matrix.

\section{Movies}

The movies referred to in the text are available in the additional materials.

C.1. See "Vid1.mp4".

C.2. See "Vid2.mp4".

Received: 5 February 2021; Accepted: 29 April 2021

Published online: 17 May 2021

\section{References}

1. Beron-Vera, F. J., Olascoaga, M. J. \& Goni, G. J. Oceanic mesoscale eddies as revealed by Lagrangian coherent structures. Geophys. Res. Lett. 35(12) (2008). 
2. Mezić, I., Loire, S., Fonoberov, V. A. \& Hogan, P. A new mixing diagnostic and Gulf oil spill movement. Science 330(6003), 486-489 (2010).

3. Nencioli, F., d'Ovidio, F., Doglioli, A. M. \& Petrenko, A. A. Surface coastal circulation patterns by in-situ detection of Lagrangian coherent structures. Geophys. Res. Lett. 38(17) (2011).

4. Olascoaga, M. J. \& Haller, G. Forecasting sudden changes in environmental pollution patterns. Proc. Natl. Acad. Sci. 109(13), 4738-4743 (2012).

5. Tang, W., Knutson, B., Mahalov, A. \& Dimitrova, R. The geometry of inertial particle mixing in urban flows, from deterministic and random displacement models. Phys. Fluids 24(6), 063302 (2012).

6. Natusch, D. F. S., Wallace, J. R. \& Evans, C. A. Toxic trace elements: Preferential concentration in respirable particles. Science 183(4121), 202-204 (1974).

7. Espinosa-Gayosso, A., Ghisalberti, M., Ivey, G. N. \& Jones, N. L. Density-ratio effects on the capture of suspended particles in aquatic systems. J. Fluid Mech. 783, 191-210 (2015).

8. Shaw, R. A., Reade, W. C., Collins, L. R. \& Verlinde, J. Preferential concentration of cloud droplets by turbulence: Effects on the early evolution of cumulus cloud droplet spectra. J. Atmos. Sci. 55(11), 1965-1976 (1998).

9. Sapsis, T. \& Haller, G. Inertial particle dynamics in a hurricane. J. Atmos. Sci. 66(8), 2481-2492 (2009).

10. Haszpra, T. \& Tél, T. Volcanic ash in the free atmosphere: A dynamical systems approach. J. Phys. Conf. Ser. 333(1), 012008 (2011).

11. Mittal, R., Ni, R. \& Seo, J. The flow physics of COVID-19. J. Fluid Mech. 894, F2 (2020).

12. Cummins, C. P., Ajayi, O. J., Mehendale, F. V., Gabl, R. \& Viola, I. M. The dispersion of spherical droplets in source-sink flows and their relevance to the COVID-19 pandemic. Phys. Fluids 32(8), 083302 (2020).

13. Verma, A. K., Bhatnagar, A., Mitra, D. \& Pandit, R. First-passage-time problem for tracers in turbulent flows applied to virus spreading. Phys. Rev. Res. 2(3), 033239 (2020).

14. Contini, D., Costabile, F. Does Air Pollution Influence COVID-19 Outbreaks? mdpi.com (2020).

15. Abuhegazy, M., Talaat, K., Anderoglu, O. \& Poroseva, S. V. Numerical investigation of aerosol transport in a classroom with relevance to COVID-19. Phys. Fluids 32(10), 103311 (2020).

16. Sapsis, T. \& Haller, G. Clustering criterion for inertial particles in two-dimensional time-periodic and three-dimensional steady flows. Chaos Interdiscip. J. Nonlinear Sci. 20(1), 017515 (2010).

17. Maxey, M. R. \& Riley, J. J. Equation of motion for a small rigid sphere in a nonuniform flow. Phys. Fluids 26(4), 883-889 (1983).

18. Bec, J. Fractal clustering of inertial particles in random flows. Phys. Fluids 15(11), L81-L84 (2003).

19. Bec, J. et al. Heavy particle concentration in turbulence at dissipative and inertial scales. Phys. Rev. Lett. 98(8), 084502 (2007).

20. Ravichandran, S. \& Govindarajan, R. Caustics and clustering in the vicinity of a vortex. Phys. Fluids 27(3), 033305 (2015).

21. Adrian, R.J., Westerweel, J. Particle Image Velocimetry. ISBN 978-0-521-44008-0 (Cambridge University Press, 2011).

22. Healy, D. P. \& Young, J. B. Full Lagrangian methods for calculating particle concentration fields in dilute gas-particle flows. Proc. R. Soc. A Math. Phys. Eng. Sci. 461(2059), 2197-2225 (2005).

23. Schmid, P. J., Li, L., Juniper, M. P. \& Pust, O. Applications of the dynamic mode decomposition. Theor. Comput. Fluid Dyn. 25(1-4), 249-259 (2011).

24. Schmid, P. J. Dynamic mode decomposition of numerical and experimental data. J. Fluid Mech. 656, 5-28 (2010).

25. Kutz, J. N., Brunton, S. L., Brunton, B. W., Proctor, J. L. Dynamic mode decomposition: Data-driven modeling of complex systems. In Society for Industrial and Applied Mathematics. ISBN: 978-1-61197-449-2 (2016).

26. Tu, J. H., Rowley, C. W., Luchtenburg, D. M., Brunton, S. L., Kutz, J. N. On Dynamic Mode Decomposition: Theory and Applications. arXiv preprint arXiv:1312.0041. (2013).

27. Young, W., Pumir, A. \& Pomeau, Y. Anomalous diffusion of tracer in convection rolls. Phys. Fluids A 1, 462 (1989).

28. Sarracino, A., Cecconi, F., Puglisi, A. \& Vulpiani, A. Nonlinear response of inertial tracers in steady laminar flows: Differential and absolute negative mobility. Phys. Rev. Lett. 117, 174501 (2016).

29. Tabeling, P. Two-dimensional turbulence: A physicist approach. Phys. Rep. 362, 1 (2002).

30. Wilkinson, M. \& Mehlig, B. Caustics in turbulent aerosols. Europhys. Lett. 71(2), 186-192 (2005).

31. Wilkinson, M., Mehlig, B. \& Bezuglyy, V. Caustic activation of rain showers. Phys. Rev. Lett. 97(4), 048501 (2006).

32. Gustavsson, K., Meneguz, E., Reeks, M. \& Mehlig, B. Inertial-particle dynamics in turbulent flows: Caustics, concentration fluctuations and random uncorrelated motion. New J. Phys. 14(11), 115017 (2012).

33. Wang, L. P., Maxey, M. R., Burton, T. D. \& Stock, D. E. Chaotic dynamics of particle dispersion in fluids. Phys. Fluids A Fluid Dyn. 4(8), 1789-1804 (1992).

34. Taira, K. et al. Modal analysis of fluid flows: An overview. AIAA J. 55(12), 4013-4041 (2017).

35. Helman, A. The Finest Peaks-Prominence and Other Mountain Measures. ISBN-10 : 141205995X (Trafford Publishing, 2005).

36. Jähne, B., Scharr, H., \& Körkel, S. Principles of filter design. In Handbook of Computer Vision and Applications. (Academic Press, 1999).

37. Shapiro, L.G., Stockman, G.C. Computer Vision 53-54. ISBN 978-0130307965 (Prentice Hall, 2001).

38. Cencini, M. et al. Dynamics and statistics of heavy particles in turbulent flows. J.Turbulence (7), N36 (2006).

39. Jayaram, R., Jie, Y., Zhao, L. \& Andersson, H. I. Clustering of inertial spheres in evolving Taylor-Green vortex flow. Phys. Fluids 32(4), $043306(2020)$.

40. Angriman, S., Mininni, P. D. \& Cobelli, P. J. Velocity and acceleration statistics in particle-laden turbulent swirling flows. Phys. Rev. Fluids 5(6), 064605 (2020).

41. Marensi, E., Yalnı, G., Hof, B., Budanur, N. B. Symmetry-Reduced Dynamic Mode Decomposition of Near-Wall Turbulence. arXiv preprint arXiv:2101.07516 (2021).

\section{Acknowledgements}

This work is supported by Board of Research in Nuclear Sciences (BRNS Sanctioned no. 39/14/05/2018-BRNS), Science and Engineering Research Board EMEQ program (SERB Sanctioned no. EEQ/2017/000164), Infosys Foundation Young Investigator Award, and IISc startup grant. We thank Rahul Pandit for valuable inputs. JKA acknowledges useful discussions with Akhilesh Kumar Verma.

\section{Author contributions}

J.K.A, S.S., and A.K. conceived the problem. O.S. and J.K.A. performed simulations. All authors analysed the results. O.S. and J.K.A. wrote the main manuscript text. All authors reviewed the manuscript.

\section{Competing interests}

The authors declare no competing interests. 


\section{Additional information}

Supplementary Information The online version contains supplementary material available at https://doi.org/ 10.1038/s41598-021-89953-3.

Correspondence and requests for materials should be addressed to J.K.A.

Reprints and permissions information is available at www.nature.com/reprints.

Publisher's note Springer Nature remains neutral with regard to jurisdictional claims in published maps and institutional affiliations.

(c) (i) Open Access This article is licensed under a Creative Commons Attribution 4.0 International License, which permits use, sharing, adaptation, distribution and reproduction in any medium or format, as long as you give appropriate credit to the original author(s) and the source, provide a link to the Creative Commons licence, and indicate if changes were made. The images or other third party material in this article are included in the article's Creative Commons licence, unless indicated otherwise in a credit line to the material. If material is not included in the article's Creative Commons licence and your intended use is not permitted by statutory regulation or exceeds the permitted use, you will need to obtain permission directly from the copyright holder. To view a copy of this licence, visit http://creativecommons.org/licenses/by/4.0/.

(C) The Author(s) 2021 